\title{
Synthesis and Characterization of Gadolinium Doped Zinc Oxide Nanorods Thin Films
}

\author{
N. Sarip ${ }^{1, a}$, A.L. Nordin ${ }^{1}$, M.S.M. Sam ${ }^{1}$, S.A. Kamaruddin ${ }^{1}$, F. Mahmud', \\ S.N.M. Tawil ${ }^{2, b}$ and M.Z. Sahdan ${ }^{1, \mathrm{c}}$ \\ ${ }^{1}$ Microelectronics and Nanotechnology-Shamsuddin Research Centre (MiNT-SRC), Universiti Tun \\ Hussein Onn Malaysia, 86400 Parit Raja, Batu Pahat, Johor, Malaysia \\ ${ }^{2}$ Department of Electrical and Electronic Engineering, \\ National Defence University of Malaysia, Kem Sg. Besi, 57000 Kuala Lumpur, Malaysia \\ anurulnadiasarip88@gmail.com, bsitinooraya@gmail.com, 'zainizno@gmail.com
}

Keywords: Zinc oxide nanorods arrays, chemical solution deposition, annealing temperature, thin films.

Abstract.ZnO nanorods (NRs) arrays were synthesized by chemical solution deposition (CSD) method on commercial glass substrate with $\mathrm{ZnO}$ thin film act as seed layer prepared by sol-gel spin coating. The effect of annealing temperature of $150^{\circ} \mathrm{C}, 250^{\circ} \mathrm{C}$ and $500^{\circ} \mathrm{C}$, respectively, on the structural growth was investigated. The observation reveals the structural improvement as the annealing temperature increased. The influence of gadolinium doping to $\mathrm{ZnO} \mathrm{NRs}$ arrays wasexplored upon the structural and optical features. The FESEM imaging along with XRD, AFM and UV-Vis analysis were conducted to dissect the information gained by performing a study case on various gadolinium doping content in the range of 1 at. $\%$ to 4 at. $\%$. Based on the results, the correlation between the doping content were drawn in details in this paper.

\section{Introduction}

Zinc oxide $(\mathrm{ZnO})$ is well known in the scientific community to possess a lot of advantages in its nature, making it as one of the most prominent material for a variety of electronic and optoelectronic applications [1]. However on the contrary, doped $\mathrm{ZnO}$ can hold some very different properties compared to that of pure $\mathrm{ZnO}$. Group III elements, i.e. $\mathrm{Ga}, \mathrm{Al}$, and $\mathrm{In}$ can be used as $n$ type dopant in $\mathrm{ZnO}$, have been generally recognized to influence the optical and also the electrical properties of the materials [2]. Other elements of rare earth group, i.e. Ce, Er, Eu, La, Tb, Tm, Yb, and Dy as $p$-type dopant to $\mathrm{ZnO}$, have also attracted significant attention among the community due to their unique optical properties which give to intense emission peaks in the visible and near infrared range [3].Hence, the electrical conductivity, type of conduction and band gap range, including the magnetic characteristics of the nanomaterial can be manipulated through doping. Therefore, doping effect can enhance the present properties of $\mathrm{ZnO}$ and provides space for new applications possible.

Presently, $\mathrm{ZnO}$-based opto-electronic devices have been reported to appear as doping of $\mathrm{ZnO}$ began to become accessible. The facile addition of metal precursor to the solution may not be as straightforward as it seems since the metal atom might not fuse in the $\mathrm{ZnO}$ structure, producing a secondary phase denoted to the existence of foreign element [4]. Fabrication of stable and devicequality $p$-type $\mathrm{ZnO}$, however, has not been realize regardless of a large number of publications stating successful demonstration of $p$-type [5, and reference therein]. Numerous reports on impurities doped with $\mathrm{ZnOhave}$ been driven to minimize the resistivity and to boost the opacity of the films [6, and reference therein]. Gadolinium (Gd) metal of the lanthanum group element was selected as dopant in the work reported here based on the the $\mathrm{Gd}_{2} \mathrm{O}_{3}$ physical properties in optical application in amendment to both the electrical and optical characteristics of the $\mathrm{ZnO}$ thin films.

This work is expected to provide the science and research information necessary towards the realization of the new enhanced functionality devices through the integration of photonic, electronic 
and magnetic properties in a single device that will be beneficial for spin light-emitting diodes (LEDs), novel microprocesses and sensitive biological and chemical sensors.Our motivation on $\mathrm{ZnO}$ thin film is, therefore, to examine the structural and optical properties obtained through a feasible, toxic free, chemical solution deposition method by manipulating the Gd doping content. Comparative studies concerning the characterization of pure and Gd-doped $\mathrm{ZnO}$ thin films were also review in this paper.

\section{Experimental Setup}

\section{A) Deposition of seed layer template}

For the start, glass substrates of $2.5 \mathrm{~cm} \mathrm{X} 2.5 \mathrm{~cm}$ in size were cleansed in ultrasonic cleaning bath in acetone for several minutes and rinsed with plenty of deionized water afterwards. Acquiring indisputable good film deposition of excellent homogeneity, quality and formation is crucial. Therefore, to certify that all the contaminants and pollutants were discard from the glass surfaces, this procedure was executed.

The deposition of seed layer was operated using the spin coater technique. The working principle of spin coater utilizes the function of high acceleration with rotary motion which governs the centrifugal force in the formation of uniform thin film on flat horizontal planar. At present, droplets of a mixture sol-gel consisting of $0.4 \mathrm{~mol}$ of zinc acetate dehydrate, ethanolamine and 2-propanol were dripped onto glass substrate and was rotated at the required accelerations whereby the spin coater willdistribute the solution thoroughly on the surface. This step was accompanied by a heat treatment of $280^{\circ} \mathrm{C}$ for three minutes in ensuring that all the residual solution was released from the coated substrate and simultaneously increase the film adhesion with the glass substrate as well. The procedure was replicated once again to attain the necessary thickness, dependent to the solution concentration and viscosity. Next, the substrates went through a pre-anneal process. The temperature varied from $150^{\circ} \mathrm{C}$ to $500^{\circ} \mathrm{C}[5]$.

\section{B) Development of $\mathrm{ZnO}$ nanorods thin films}

Immerse solutions consisting of a mixing of $0.1097 \mathrm{~g}$ of Zinc acetate dehydrate, $0.03 \mathrm{ml}$ of ethanolamine, $50.00 \mathrm{ml}$ of deionized water and an equivalent amount of gadolinium (III) nitrate hexahydrate with respect to the doping percentile were prepared. Seeded substrates were placed in the prepared solution for the next three remaining hours at constant temperature of $95^{\circ} \mathrm{C}$. The coated substrates were then rinsed and dried at room condition. Annealing of the grown $\mathrm{ZnO}$ nanorods thin films were done at respective pre-annealing temperature for another one hour in a carbolite furnace. The desired samples were then ready to be examined under the characterization tools necessary.

\section{Results and Discussion}

In general, this section reports on the results of the synthesized Gd-doped and pure $\mathrm{ZnO}$ nanorods thin films. Analysis reports attained through all four characterization tools, namely the field emission scanning electron microscope (FE-SEM), X-Ray diffractometer (XRD), atomic force microscope (AFM) and ultraviolet - visible spectrophotometer (UV-Vis), were discussed.

\section{A) Structural properties of $\mathrm{ZnO}$ nanorods thin films}

The investigation on the fabrication of pure $\mathrm{ZnO}$ nanorods thin films at different temperature parameter conducted explores a significant impact on the structural morphology. Fig. 1 represents the diffractogram of an XRD analysis. Appearance of the following peaks of $\left(\begin{array}{lll}1 & 0 & 0\end{array}\right),\left(\begin{array}{lll}0 & 0 & 2\end{array}\right),\left(\begin{array}{lll}1 & 0 & 1\end{array}\right)$,

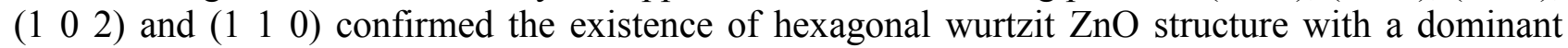
orientational growth on the $c$-axis. As seen from the figure, it is evident that $\mathrm{ZnO}$ nanorods film corresponds to (a) recorded the sharpest symmetrical peaks distribution as compared to (b) and (c). This statement is supported by the FE-SEM images as portrayed in Fig. 2. 
The films show an improvement to the surface morphology as the annealing temperature increased. Fig. 2(a) demonstrates a dense uniform grain distribution with smaller grain size. In contrast to Fig. 2(a), Fig. 2(b) and Fig. 2(c) result in larger grain gap with inconsistency in size and surface distribution. The grain size and film distribution can be verified through the AFM analysis as depicted in the Fig. 2 insets. From there, we proved that the increasein annealing temperature had resulted in reduced roughness values from $12.81 \mathrm{~nm}$ to $6.04 \mathrm{~nm}$ for $\mathrm{ZnO}$ nanorods films annealed at $150^{\circ} \mathrm{C}$ and $500^{\circ} \mathrm{C}$, respectively. Hence, the annealing temperature of $500^{\circ} \mathrm{C}$ has been selected as the optimum temperature for $\mathrm{ZnO}$ nanorods film growth.

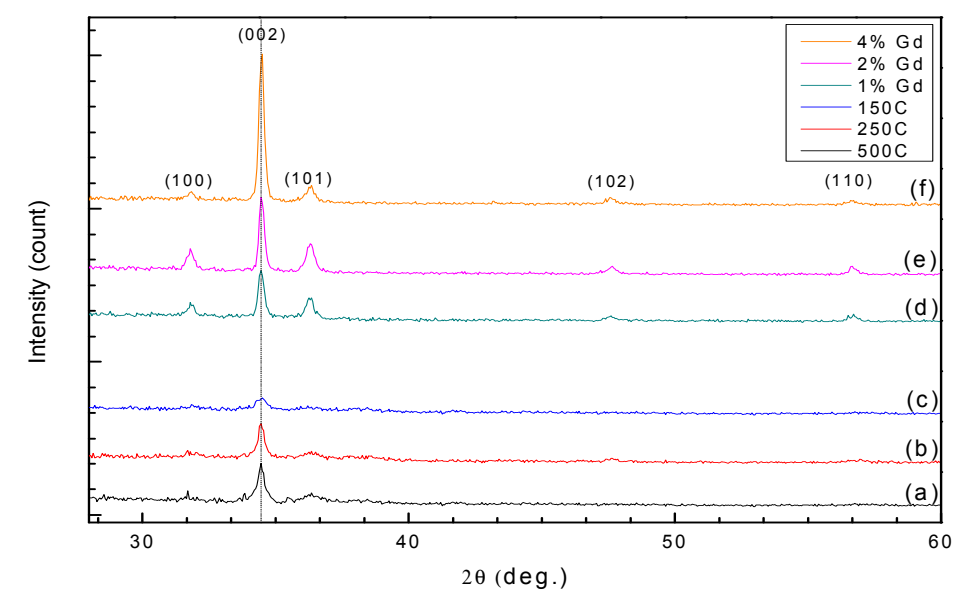

Fig. $1 \mathrm{X}$-ray diffractogram patterns of the $\mathrm{ZnO}$ nanorods thin films grown at various annealing temperature and Gd concentration.

From the observation in Fig. 2(d-f) insets, the morphological differences can be observed by performing an AFM study on different Gd concentration of 1 at. $\%, 2$ at. $\%$, and 4 at. $\%$, correspondingly. The descending roughness readings occurbecause of the temperature and the doping content of $\mathrm{Gd}$ which resulted in smoother and denser surface. These images show a noticeable variation on the homogeneity of the films with large nanorods formation. The presence of diffraction peaks indicates that the films were polycrystalline in nature with preferential orientation growth along the $\left(\begin{array}{lll}0 & 0 & 2\end{array}\right)$ plane. The XRD diffractogram illustrated in Fig. 1 also proved no formation of other phase in the samples. From FE-SEM images as pictured in Fig. 2, it was observed that the average particle size increased with the increased in doping concentrations. 


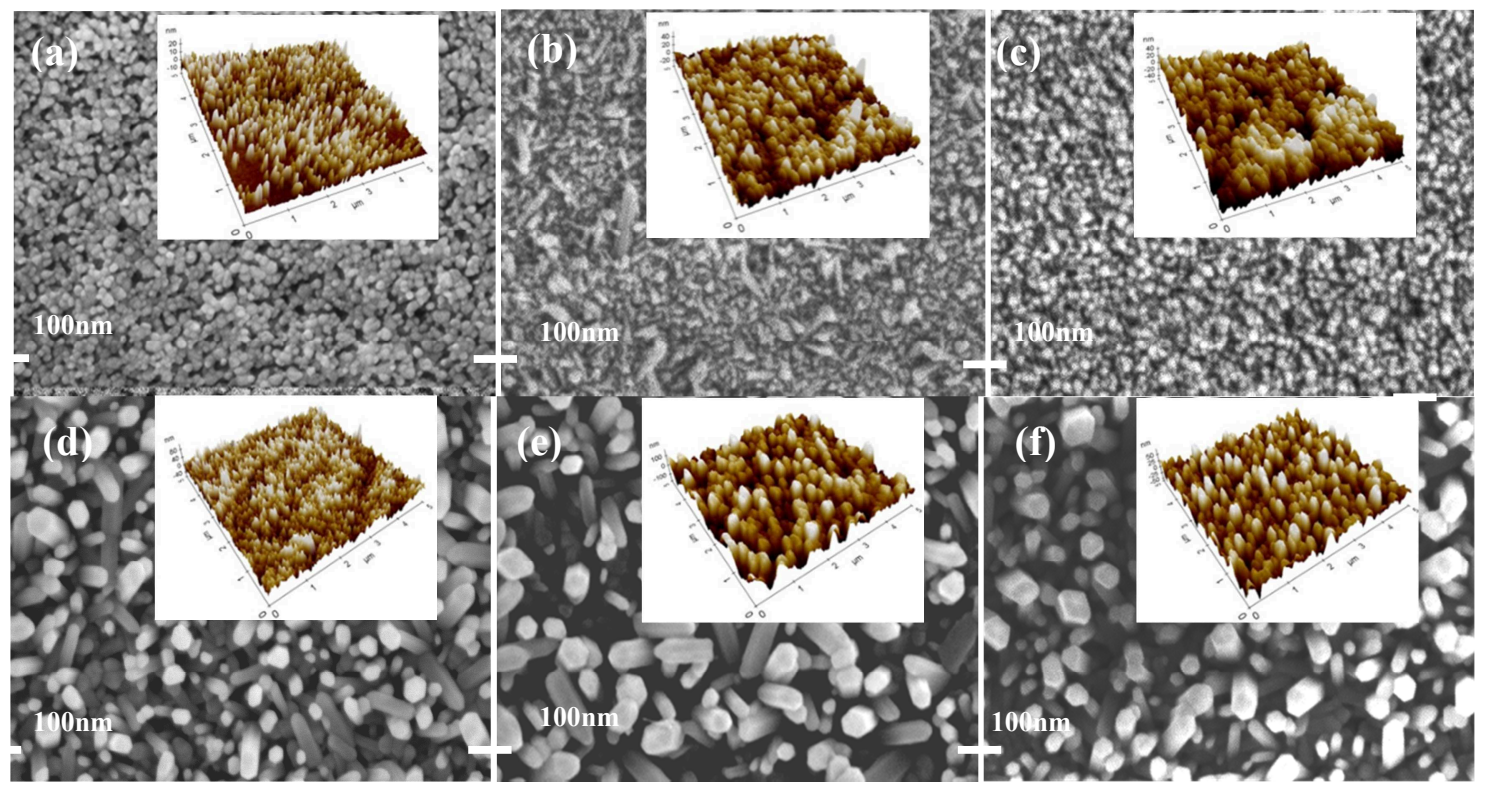

Fig. 2FESEM images of $\mathrm{ZnO}$ thin films attained at various annealing temperature (a) $500{ }^{\circ} \mathrm{C}$, (b) $250{ }^{\circ} \mathrm{C}$, (c) $150{ }^{\circ} \mathrm{C}$; and at various Gd impurity concentrations (d) 1 at. \%, (e) 2 at. \%, and (f) 4 at. $\%$, respectively. (Insets were the topographical image attained from AFM analysis of the corresponding samples.)

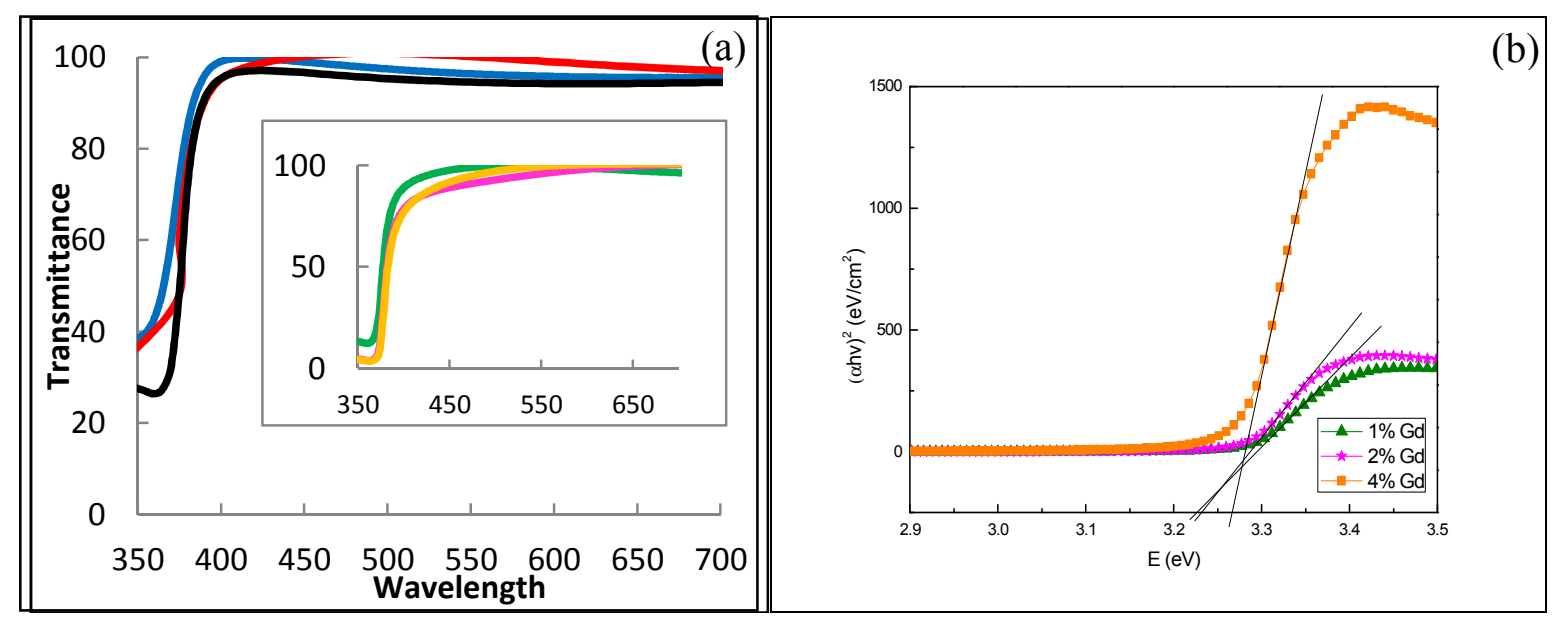

Fig. 3 (a) Graph depicted the transmittance spectra of the corresponding samples; and (b) plot of $\left(\alpha v^{2}\right)$ as a function of photon energy against for Gd-doped $\mathrm{ZnO}$ thin films.

\section{B) Optical properties of pure and $\mathrm{Gd}$-doped $\mathrm{ZnO}$ nanorods thin films}

Fig. 3 shows the transmittance and band gap of pure and Gd-doped $\mathrm{ZnO}$ nanorods thin films. From the transmittance graph, it shows that the visibile transparency is above $80 \%$ in average. It was likely to see that the visible transparency of $500{ }^{\circ} \mathrm{C}$ was more excellent compared to that of $150{ }^{\circ} \mathrm{C}$ and $250{ }^{\circ} \mathrm{C}$ samples. Therefore, from the optical analysis, the optimum temperature of $500{ }^{\circ} \mathrm{C}$ is suitable to be chosen. We could also been able to identify that the transmittance of Gd-doped $\mathrm{ZnO}$ films responded positively to the increased doping concentration of $4 \mathrm{at}$. $\% \mathrm{Gd}$ as oppose to that of 1 at. $\%$ and 2 at. $\% \mathrm{Gd}$ concentrations. The absorption analysis, on the other hand, has been increasing from 2 and 4 at. $\% \mathrm{Gd}$, whistle at 1 at. \% $\mathrm{Gd}$ the absorption decreased.The optical band gap was found to be $3.22 \mathrm{eV}$ and $3.23 \mathrm{eV}$ for $\mathrm{Gd}$-doped $\mathrm{ZnO}$ thin films at 1 at. \% and 2 at. \% $\mathrm{Gd}$ concentrations and it increased to $3.27 \mathrm{eV}$ for 4 at. \% $\mathrm{Gd}$ doping. 


\section{Conclusion}

The optimum temperature of two steps annealing of $500{ }^{\circ} \mathrm{C}$ has been selected as the optimum temperature for the doping of gadolinium to $\mathrm{ZnO}$ nanorods thin film samples. The role of Gd-doped $\mathrm{ZnO}$ can be clearly seen throughout the increase of doping concentrations. These also provided evidence that resulted in decreasing of roughness values as measured from AFM. This was the resultant of the temperature and the doping of Gd element, which has been causing the molecular structure of the hexagonal wurtzit to be deformed through the replacement of Gd atom on the $\mathrm{Zn}$ site through substitutional doping and hence resulting in a more smooth and dense upper surface of the films. The FESEM images depicted the increased in size and compact growth of the nanorods structurewith respect to the increased in Gd concentration. The optical analysisas a measure of the visible transparency reported that 4 at. \% of Gd concentration to be the best followed by 2 at. $\%$ and 1 at. \%was observed. Meanwhile for the absorbance, the absorption of light has been increasing from 2 to 4 at. $\% \mathrm{Gd}$ concentration, while at 1 at. $\%$, the absorption decreased.

\section{Acknowledgement}

The authors gratefully acknowledge The Ministry of Higher Education Malaysia for the financial support through Exploratory Research Grant Scheme (E001) and Fundamental Research Grant Scheme (FRGS2013/SG06/UPNM/02/1/1/). A special thanks to MiNT-SRC of Universiti Tun Hussein Onn Malaysia (UTHM) for such equipments provided.

\section{References}

[1] Z.L. Wang, Chinese Sci Bull 54, 4021 (2009).

[2] Z. Lin, S. Guangjie, S. Shitao, Q. Xiujuan, and H. Sihuizhi,Rare Metals30, 175 (2011).

[3] R. Zamiri, A.F. Lemos, A. Reblo, H.A. Ahangar, and J.M.F. Ferreira, Ceramic International40,532 (2014).

[4] A. Chandra Bose, and N. Rajeswari Yogamalar,Prog. in Nanotechnology and Nanomaterials2, $1(2013)$.

[5] H.-C. Cheng, C.-F. Chen, C.-Y. Tsay and J.-P. Leu, Journal of Alloys and Compounds475, L46 (2009). 